\title{
Vécu Psychologique De La Parturiente En Salle D'accouchement À La Maternité Du Centre Hospitalier Départemental Et Universitaire Du Borgou À Parakou (BENIN)
}

\section{Achille Awadé Afoukou Obossou}

UER en gynécologie Obstétrique, Faculté de Médecine,

Université de Parakou (Bénin)

\section{Kabibou Salifou}

UER en gynécologie Obstétrique, Faculté de Médecine,

Université de Parakou (Bénin)

Moufalilou Aboubakar

UER en Gynécologie Obstétrique, Faculté des sciences de la Santé,

Université d'Abomey Calavi

Fanny Maryline Nouessèwa Hounkponou Ahouingnan

UER en gynécologie Obstétrique, Faculté de Médecine, Université de

Parakou (Bénin)

Angéline Josiane Tonato Bagnan

\section{Benjamin Ignace Bodounrin Hounkpatin}

UER en Gynécologie Obstétrique, Faculté des sciences de la Santé, Université d'Abomey Calavi (Bénin)

\section{Rachidi Imorou Sidi Mahublo Vinadou Vodouhe}

\section{Landry Araye}

UER en gynécologie Obstétrique, Faculté de Médecine, Université de Parakou (Bénin)

René Xavier Perrin

UER en Gynécologie Obstétrique, Faculté des sciences de la Santé, Université d'Abomey Calavi (Bénin)

Abstract

Objective: To study parturient women's psychological real-life experience in the delivery room of CHUD-B maternity hospital. Patients and Methods: This study shows a descriptive cross-sectional study with an analytical aim and a prospective data collection. It involves 100 parturient 
women who had a vaginal delivery at the gynecology and obstetrics department of Departmental University Teaching Hospital of Borgou. It covered the period from June $11^{\text {th }}$ to August $11^{\text {th }} 2014$. Results: The average age of the parturient women was 27 years old \pm 5.55 . The parturient women were predominantly Muslims (58\%), married (48\%), and out-of-school (36\%). They had a monogamous relationship (77\%) with their husbands $(66 \%)$. Also, they had conflict with the people around them $(22 \%)$. They were anxious (58\%), distressed (27\%), and timorous and restless (57\%). The main reasons for stress noticed among these parturient women were related to the fear of stillbirth (82\%), a malformed child $(76 \%)$, an infected newborn baby $(76 \%)$, obstetrical trauma in the newborn baby $(58 \%)$, and the newborn baby's sex (26\%). Subsequently, the fears of the parturient women were: the cesarean section (64\%), maternal death (58\%), the pain of childbirth $(48 \%)$, traumatic maternal injury (47\%), post-partum hemorrhage $(45 \%)$, and the inability to face childbirth $(31 \%)$. The parturient women before getting into the delivery room confided in traditional religious authorities $(36 \%)$ who were either healers or marabous, witch doctors, spiritual advisors, or Christian priests. Conclusion: Delivery causes anxiety among parturient women whose apprehensions were about the pain of childbirth, the unborn baby, and their own mental ability to overcome the trial.

Keywords: Parturient women, real-life experience, psychology, delivery room

\section{Resume}

Objectif: étudier le vécu psychologique des parturientes en salle d'accouchement à la maternité du CHUD-B. Patientes et Méthodes: Il s'est agit d'une étude transversale, descriptive à visée analytique avec recueil prospectif auprès de 100 parturientes ayant accouché par voie basse, dans le service de gynécologie et d'obstétrique du Centre Hospitalier Universitaire Départemental du Borgou (CHUD-B) à Parakou. Elle a couvert la période du 11 Juin au 11Aout 2014. Résultats: La moyenne d'âge des parturientes était de 27 ans $\pm 5,55$. Nos patientes étaient à préominance musulmanes (58\%), mariées $(48 \%)$, non scolarisées $(36 \%)$. Elles étaient dans un foyer monogame (77\%) avec leur mari $(66 \%)$ et étaient en conflit avec l'entourage $(22 \%)$. Elles étaient anxieuses (58\%), angoissées $(27 \%)$, craintives et agitées (57\%). Les principaux motifs de stress retrouvés chez ces parturientes portaient sur la peur d'un mort né $(82 \%)$, un enfant malformé $(76 \%)$, un nouveau né infecté (76\%), des traumatismes obstétricaux chez le nouveau né $(58 \%)$, le sexe du nouveau né $(26 \%)$. Les peurs concernant les parturientes elles-mêmes étaient: la césarienne (64\%), le décès maternel $(58 \%)$, la douleur de l'enfantement (48\%), les lésions traumatiques maternelles (47\%), 
l'hémorragie de la délivrance (45\%), l'incapacité d'affronter l'accouchement (31\%). Les parturientes avant leur entrée en salle d'accouchement se confiaient aux autorités religieuses traditionnelles (36\%) qui étaient soient guérisseurs, marabouts, féticheurs, conseillers spirituels, prêtres chrétiens. Conclusion: L'accouchement génère une angoisse chez les parturientes dont les appréhensions portent sur la douleur de l'enfantement, la santé du bébé à naître et leur propre capacité mentale à surmonter l'épreuve.

Palabras-claves: Parturientes, vécu, psychologie, salle d'accouchement

\section{Introduction}

La naissance d'un enfant est un évènement merveilleux mais bouleversant sur le plan émotionnel. Elle est aussi décrite comme un évènement potentiellement stressant dans la vie de la femme (Ford \& Ayers, 2008).

En effet, pour Szejer et al. (1994); les parturientes peuvent éprouver des sentiments positifs : la sensation de plénitude, d'épanouissement voire même la sensation de plaisir lors de l'accouchement. Il existe aussi des sentiments négatifs : anxiété, angoisse, panique, peur face à certaines situations de la vie qui peuvent survenir durant l'accouchement comme la douleur, la mort de l'enfant et ou de la mère (Szejer et al., 1994).

La salle d'accouchement est un milieu aliénant pour de nombreuses femmes où les routines institutionnelles telles que le déshabillage systématique dès l'arrivée, le manque d'intimité, vont déposséder certaines parturientes de leur sentiment d'intimité (Creedy, Shocket \& Horsfall, 2000).

L'anxiété provoquée par la perte de leur sentiment de contrôle, peut gêner le déroulement normal du travail (Creedy, Shocket \& Horsfall, 2000). Simkin (1990) rapporte que le sentiment de la parturiente d'avoir ou non le contrôle détermine le souvenir positif ou négatif de la naissance. De même, l'issue physique et émotionnelle du travail est bien meilleure si la femme participe aux prises de décision (Olde et al., 2006). Wijma et al. (2001) avaient fait le lien entre l'anxiété et l'expression des différentes peurs. Ainsi, selon leur étude; $79,2 \%$ des nullipares avaient un niveau de peur élevé dont $55,4 \%$ étaient anxieuses. De même chez les multipares; $77,4 \%$ avaient un niveau élevé de peur et $57,9 \%$ étaient anxieuses.

En 2012, Blondeau avait retrouvé dans une maternité à Bordeaux en France que 57,5\% des parturientes étaient anxieuses ou angoissées, 35,5\% avaient peur de la douleur, $40 \%$ avaient peur d'accoucher un mort-né, 17\% avaient peur de la malformation, $25 \%$ avaient peur de la mort, $37,5 \%$ avaient peur de l'imprévu durant l'accouchement.

En Afrique, une étude sénégalaise (Royer, 1998) comparant l'accouchement traditionnel et l'accouchement moderne avait conclu que les 
parturientes accouchant traditionnellement étaient bien entourées par leur mère, leurs sœurs, leurs cousines qui s'occupent d'elles. Ces parturientes étaient moins angoissées que celles qui avaient effectué un accouchement assisté par une sage-femme considérée personne étrangère à leurs yeux alors qu'aucune assistance de leur proche n'était pas autorisée.

Dans nos sociétés africaines, des rites, des prières, des sacrifices et des consultations d'oracle entourent les grands événements comme l'accouchement d'une femme. Ces pratiques rassurent l'individu et les familles puis réduisent l'inquiétude, l'anxiété et les différentes peurs liées à l'accouchement (Jacques, 2008).

Le bien être psychologique de la parturiente en salle d'accouchement, sa participation, jouent un grand rôle dans le bon déroulement du travail d'accouchement, dans la prévention des psychoses puerpérales et des états de stress post traumatiques de la maternité (Olde et al., 2006). Au Bénin, encore moins dans sa partie septentrionale, aucune étude n'a renseigné sur le vécu psychologique de la parturiente en salle d'accouchement. C'est cela qui justifie notre intérêt pour ce sujet dont l'objectif était d'étudier le vécu psychologique et les facteurs déterminant les manifestations psychologiques des parturientes en salle d'accouchement à la maternité du CHUD-B.

\section{CADRE, PATIENTES ET METHODES D'ETUDE}

Notre étude s'est déroulée dans le service de gynécologie obstétrique du Centre Hospitalier Universitaire départemental du Borgou (CHUD/B), le seul hôpital universitaire de référence du Nord Bénin.

Il s'était agi d'une étude transversale à visée descriptive et analytique avec recueil prospectif des données. Elle a été réalisée sur une période de deux mois allant du 11 Juin au 11 Août 2014. Elle a portée sur toutes les parturientes ayant accouché à terme par voie basse à la maternité du CHUD-Borgou durant la période de l'étude. Ont été incluses, toutes accouchées par voie basse à terme ayant donné leur consentement quelque soit l'état du nouveau-né à la naissance. Les parturientes ayant refusé de participer à l'étude, chez qui le diagnostic de mort fotal in utero a été pose avant l'entrer en salle d'accouchement et celles ayant accouché à domicile ou referées ont été exclues.

Nous avions fait un recrutement exhaustif de toutes les parturientes ayant accouché à terme durant la période de l'étude.

La variable dépendante était représentée par le vécu psychologique de la parturiente en salle d'accouchement.

Les variables indépendantes étaient en rapport avec les:

-Les caractéristiques sociodémographiques (l'âge, la profession, la situation matrimoniale, le niveau d'instruction, le niveau socio- 
économique, la religion, l'enquête sociale de la parturiente, et personne vivant avec la parturiente).

-Les données relatives à l'état psychologique de la parturiente (la perception de l'accouchement, les informations attendues sur l'accouchement, les informations reçues par la parturiente sur la salle d'accouchement, la traduction de l'information reçue de la sage-femme ou du gynécologue, les craintes en rapport avec l'accouchement, l'état d'esprit en salle d'accouchement, les différentes peurs par rapport à l'accouchement, l'anxiété et l'angoisse en salle d'accouchement).

-Les données socio-anthropologiques (l'état des relations sociales avec l'entourage familial, le milieu professionnel, la communauté, l'existence de confidents spirituels (prêtre, marabout, féticheur, guérisseur, centre spirituel, les pratiques mystico-religieuses courantes avant l'accouchement et les pratiques mystico-religieuses courantes de la parturiente).

Les données ont été organisées, traitées, analysées à l'aide du logiciel SPSS17.

\section{Resultats}

Durant la période de l'étude 113 parturientes avaient accouché par voie basse à terme à la maternité du CHUD-B. Parmi ces parturientes 13 avaient refusé de participer à l'étude. Le taux de participation a été donc de $88 \%$.

\section{CARACTERISTIQUES PARTURIENTES \\ SOCIODEMOGRAPHIQUES DES}

\section{- L'âge}

La moyenne d'âge était de 27 ans avec un écart type de 5,55. L'âge minimal et l'âge maximal étaient respectivement de 16 ans et de 41 ans. La majorité des parturientes enquêtées soit 70\%, appartenaient à la tranche d'âge de 20 à 29 ans. Les tranches d'âge étaient pour les moins de 19 ans : 7\%, 20 à 24 ans : 29 ans, 25 à 29 ans : 41 \%, 30 à 34 ans $11 \%$ et au-delà de 35 ans, 12\%.

\section{- Niveau d'étude, situation matrimoniale, secteur d'activité professionnelle et religion}

Les parturientes qui n'avaient aucun niveau d'instruction représentaient $36 \%$ de l'effectif total. Une(1) parturiente sur 2 était mariée. Ces informations sont résumées dans le tableau I.

Tableau I. Répartition des parturientes enquêtées sur leur vécu psychologique de la salle d'accouchement à Parakou au CHUD-B en 2014 selon le niveau d'étude, la situation matrimoniale, le secteur d'activité professionnelle et la religión 
Non scolarisé

Niveau d'étude

Primaire

Secondaire

20,0

Supérieur

Situation matrimoniale

Mariée

Concubinage

Célibataire

Divorcée/séparée

Secteur d'activité professionnelle de la parturiente

Commerce et vente

Artisanes

Agents publics ou privés

Sans activité (élèves, étudiantes)

Ouvrière, manœuvre non agricole

Traditionnelle

\section{Religion}

Islamique

Catholique

Protestants

\section{- Niveau socioéconomique, profession du mari et personne avec qui vit la parturiente}

Sur 10 parturientes, 7 vivaient avec leur mari et de 2 avec les beauxparents. Le niveau de vie socioéconomique était bas à $53 \%$.Ces informations sont résumées dans le tableau II.

Tableau II. Répartition des parturientes enquêtées sur leur vécu psychologique de la salle d'accouchement à Parakou au CHUD-B en 2014 selon le niveau socioéconomique, la profession du mari et la personne avec qui vit la parturiente

\begin{tabular}{rr}
\hline Effectif & Pourcentage (\%) \\
\hline
\end{tabular}

Niveau socioéconomique

Bas

Elevé

Profession du mari

Salariés 
Commerçants

Agriculteurs, éleveurs, pêcheurs

Sans activité

Type de foyer

Monogame

Polygame

Personne avec qui réside la parturiente

Mari

\section{- Enquête sociale}

Nous avions dénombré 78 patientes (78\%) n'ayant aucun conflit avec leur entourage, $13(13 \%)$ en conflit avec leur mari, $6(6 \%)$ en conflit avec leur belle mère et $3(3 \%)$ en conflit avec d'autres personnes en dehors de leur famille.

\section{MANIFESTATIONS PSYCHOLOGIQUES}

\section{- L'anxiété et l'angoisse}

Plus de la moitié des parturientes soit 58\% affirmaient être anxieuses par rapport à l'accouchement et $27 \%$ étaient angoissées.

La répartition des parturientes enquêtées sur leur vécu psychologique de la salle d'accouchement au CHUD-B en 2014 selon leur anxiété et leur angoisse est représentée sur les figures $\mathrm{n}^{\circ} 1 \mathrm{a}$ et $1 \mathrm{~b}$.
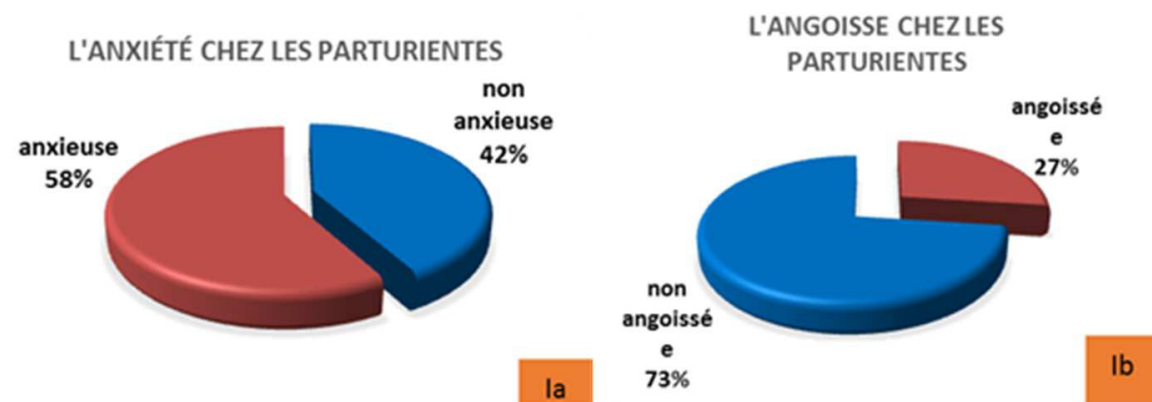

$\underline{\text { Figure } \mathbf{n}^{\circ} \mathbf{1}}$. Répartition des parturientes enquêtées sur leur vécu psychologique de la salle d'accouchement Parakou à au CHUD-B en 2014 selon leur anxiété et leur angoisse

- Etat d'esprit des parturientes à leur arrivée en salle de naissance et motifs de peur

\section{$\checkmark$ Etat d'esprit des parturientes en salle de naissance}

La moitié des parturientes 50\% avaient manifesté de la crainte 7 (7\%) avaient manifesté de l'agitation à leur arrivée en salle de naissance. Par contre, 43 restantes (43\%) étaient sereines. 


\section{$\checkmark$ Motifs de peur des parturientes en salle de naissance}

Les trois principaux objets de peur chez les parturientes enquêtées portaient globalement sur l'état de santé du bébé (mort-né $(82 \%)$; enfant malformé (76\%); infection du nouveau-né (76\%)). La répartition des parturientes enquêtées sur leur vécu psychologique de la salle d'accouchement au CHUD-B en 2014 selon les motifs de peur est représentée sur la Figure ${ }^{\circ} 2$.

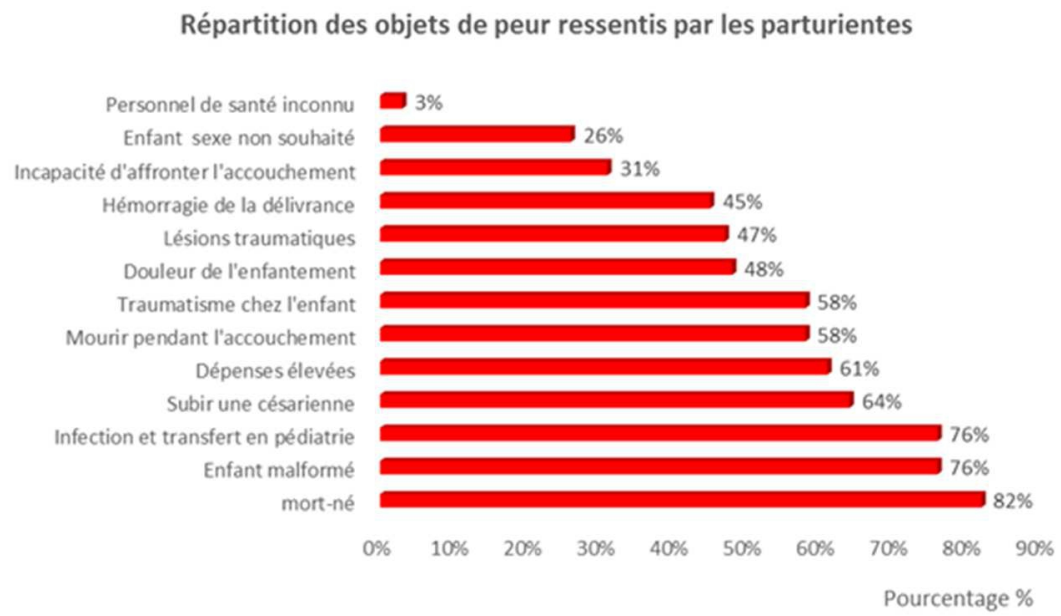

Figure n 2. Répartition des parturientes enquêtées sur leur vécu psychologique de la salle d'accouchement à Parakou au CHUD-B en 2014 selon les motifs de peur

\section{REPRESENTATIONS CULTURELLES DE L'ACCOUCHEMENT}

- Modes de préparation des parturientes pour l'accouchement: type d'autorité religieuse à qui les parturientes se sont confiées

Sur les 100 parturientes enquêtées, $36(36 \%)$ d'entre elles s'étaient confiées à une autorité religieuse traditionnelle ou moderne avant d'aller en salle d'accouchement.

Ces informations sont représentées sur la figure numéro 3:

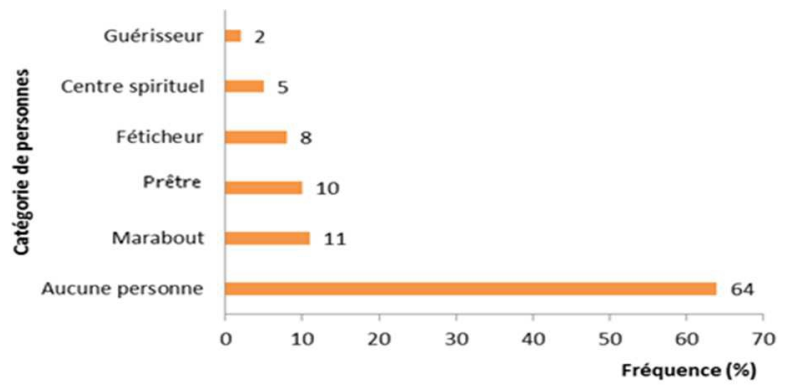

Figure n⿳03. Répartition des parturientes enquêtées sur leur vécu psychologique de la salle d'accouchement à Parakou au CHUD-B en 2014 selon les autorités à qui elles s'étaient confiées 


\section{- Conseils reçus et attitudes des parturientes face aux conseils}

Les conseils ou soutiens reçus étaient d'ordre spirituel ou religieux. Il s'agissait de prières $(63 \%)$ et de sacrifices $(26 \%)$. Ces conseils ont été suivis par les parturientes enquêtées qui en avaient bénéficié à $98 \%$. Les raisons principales de respect de ces conseils étaient: la foi personnelle $(54 \%)$, l'exigence religieuse $(16 \%)$ et les coutumes ou exigences familiales $(23 \%)$.

Le tableau III ci-dessous regroupe les conseils et attitudes des parturientes face aux conseils reçus.

Tableau III. Répartition des parturientes enquêtées sur leur vécu psychologique de la salle d'accouchement à Parakou au CHUD-B en 2014 suivant les conseils reçus et leurs attitudes face à ces conseils

\begin{tabular}{lrr}
\hline & Effectif & Pourcentage(\%) \\
\hline Conseils ou soutiens reçus: Sacrifices & 50 & \\
Oui & 30 & 62,5 \\
Non & & 37,5 \\
$\quad$ Conseils ou soutiens reçus: Prières & 21 & 26,3 \\
Oui & 59 & 73,8 \\
Non & & \\
Respect des conseils & 61 & 98,0 \\
Oui & 1 & 2,0 \\
Non & & \\
Raisons de respect des conseils & 10 & 16,0 \\
Exigence religieuse & 14 & 23,0 \\
Coutumes et exigences familial & 4 & 7,0 \\
Conseils d'un ami & 33 & 54,0 \\
Croyance personnelle & & 14,0 \\
Consultation d'oracle & 14 & 86,0 \\
Oui & 86 & 14,0 \\
Non & & 86,0 \\
Accomplissement de rituel & 14 & \\
Non & 86 & \\
& &
\end{tabular}

\section{Discussion}

\section{CARACTERISTIQUES SOCIODEMOGRAPHIQUES DES} PARTURIENTES

L'âge moyen des parturientes au cours de notre étude était de 27 ans. L'âge moyen est variable dans la littérature comme le témoignent les travaux de Blondeau et al. (2012) en France en 2012 (26,7 ans); Cavalho et al. (2008) au Brésil (24ans); Ford et Ayers (2001) aux USA en 2008(32ans). L'âge moyen de notre étude pourrait s'expliquer par le fait que la plupart des parturientes enquêtées $65 \%$ étaient au moins à leur deuxième accouchement.

Dans notre série, $36 \%$ de nos parturientes n'ont jamais été scolarisées ; 20\% n'ont acquis que l'enseignement primaire. Ce résultat 
s'explique par le faible taux de scolarisation dans les pays sous-développés en général mais aussi le Nord-Bénin est la partie du pays ayant un taux national de scolarisation le plus bas (PNUD, 2009). Notre résultat est largement supérieur à celui trouvé par Musa et al. (2005) en 2008 au Nigeria qui est de $12,4 \%$.

Dans notre échantillon, la majorité des parturientes menaient une activité à faible revenu (ménagère, élève, ouvrière, artisane, agricultrice, et revendeuse) soit $85 \%$. Cette prédominance de parturientes menant une activité à faible revenu pourrait expliquer les 53\% de niveau économique bas et les $35 \%$ de niveau économique moyen retrouvé durant l'étude. Ces résultats mettent en évidence les difficultés financières auxquelles sont confrontées les populations d'Afrique et en particulier celles du Bénin pour faire face à leur problème de santé. Le même constat a été fait par Musa et al. (2005) au Nigeria en 2008. Ceci traduit le bas niveau de vie de la population africaine surtout, l'Afrique sub-saharienne. Contrairement à l'Afrique sub-saharienne, le niveau de vie des parturientes est élevé dans les pays développés, confirmé par Blondeau et al. (2012) en France avec un faible taux d'activité de faible revenue des parturientes à $7,5 \%$.

\section{LES REPRESENTATIONS CULTURELLES DE L'ACCOUCHEMENT CHEZ LES PARTURIENTES}

En ce qui concerne les représentations culturelles de l'accouchement, $36 \%$ des parturientes enquêtées s'étaient confiées à une autorité religieuse traditionnelle ou moderne (marabout, guérisseur, féticheur, centre spirituel, prêtre). Les conseils ou soutiens reçus par les parturientes de la part de ces autorités religieuses étaient les prières, les sacrifices, la consultation d'oracle, l'accomplissement de rituel. Royer (1998) au Sénégal, avaient retrouvé un résultat similaire avec $40 \%$ des parturientes qui s'étaient confiées à une autorité religieuse traditionnelle ou moderne avant d'aller en salle d'accouchement. Ces résultats justifient le rôle important des autorités religieuses dans nos sociétés africaines. Aussi l'Afrique se particularise-t-elle avec la foi aux sciences occultes où des rites, des prières, des sacrifices et des consultations d'oracle entourent les grands événements et l'accouchement d'une femme n'y échappe pas.

\section{LES MANIFESTATIONS PSYCHOLOGIQUES DES PARTURIENTES EN SALLE D'ACCOUCHEMENT \\ L'anxiété et l'angoisse}

Dans notre série près de 6 parturientes sur 10 avaient affirmé être anxieuses et $23 \%$ avaient avoué être angoissées par rapport à 1'accouchement. Nos résultats sont similaires à ceux retrouvés par Ford et al. (2008) aux USA qui avaient trouvé 61\% de parturientes anxieuses par 
rapport à l'accouchement et Blondeau et al. (2012) en France qui avaient publié 57,5\% parturientes anxieuses par rapport à l'accouchement au terme de leur étude. Ces résultats traduisent que l'anxiété et l'angoisse en rapport avec l'accouchement sont indépendantes du niveau de développement et par conséquent de la qualité des infrastructures hospitalières.

\section{Etat d'esprit des parturientes en salle de naissance}

Dans notre étude plus de la moitié, soit 57\% des parturientes avaient manifesté de la crainte voire de l'agitation à leur arrivée en salle de naissance tandis que $43 \%$ des parturientes étaient sereines. Ceci pourrait s'expliquer par l'absence de préparation à l'accouchement des parturientes avant l'accouchement. Blondeau et al. (2012) en France avaient trouvé quant à eux $37 \%$ de parturientes craintives et $63 \%$ sereines par rapport à l'accouchement. Le taux élevé de parturientes sereines par rapport au nôtre pourrait s'expliquer par le fait qu'en France il existe presque toujours des séances de préparation à l'accouchement dirigées par des équipes rompues à la tâche dans ce domaine. L'analgésie péridurale étant toujours disponible en salle d'accouchement.

\section{Les objets de peur des parturientes en salle de naissance}

Les taux élevés de parturientes dans notre étude ayant manifesté des peurs par rapport à l'état de santé du nouveau-né $(76 \%)$, l'état maternel (64\%), et au coût élevé des soins (61\%) pourraient s'expliquer par l'inexistence de préparation psychologique des gestantes pour l'accouchement et le faible niveau économique qui fait craindre aux familles les coûts des prestations. Blondeau et al. (2012) en France avaient trouvé comme objets de peur en rapport avec l'état de santé du nouveau-né et l'état maternel, des pourcentages nettement inférieurs au notre (peur d'accoucher un mort-né $(40 \%)$, peur du décès maternel durant l'accouchement $(25 \%)$, peur de la césarienne (23\%), peur de la malformation du nouveau-né (17\%)).

\section{Conclusion}

Rien que la perspective d'accoucher dans une maternité qui a ses rituels propres intensifie l'angoisse des parturientes chez qui les appréhensions portent sur la douleur de l'enfantement, la santé du bébé à naître et leur propre capacité mentale à surmonter l'épreuve.

\section{References:}

1. Blondeau M. (2012). Vécu de la salle de naissance par la parturiente appartenant à une profession de santé. Mémoire de fin d'études de sage-femme : Université Victor Segalen-Bordeaux2; 2012. 
Disponible sur url : $\underline{\text { https://dumas.ccsd.cnrs.fr/dumas- }}$ 00716839/document.

2. Carvalho AEV, Martinez FE, \& Linhares MBM (2008). Maternal Anxiety and Depression and Development of Prematurely Born Infants in the First Year of Life. The Span J Psychol.; 11(2):600-8.

3. Creedy DK, Shocket IM, \& Horsfall I. (2000). Child birth and the development of acute trauma symptoms, incidence and contributing factors. Birth; 27(2):104-11.

4. Ford E. \& Ayers S. (2008). Stressful events and support during birth: The effect on anxiety, mood and perceived control. Journal of Anxiety Discorders; 23:260-8.

5. Jacques B (2008). La sociologie de l'accouchement. Sciences sociales et santé ; $26 \underline{(1)}$ : 124-7

6. Musa O I, Akande T M, Adebayo V T. (2005). Stressful events experience by mothers with children on admission at the emergency pediatric unit of a tertiary hospital in northern Nigeria. European Journal of scientific Research; 10(3):66-70.

7. Olde E, Van Der Hart O, Kleber R et al. (2006). Post-traumatic stress following childbirth. Clinical Psychology review; 27(2):26 -31.

8. Programme des Nations Unies pour le Développement (2009). Les objectifs du millénaire pour le developpement: Situations et perspectives. PNUD; Juillet: 36p. Disponible sur URL : http://www.undp.org/content/dam/benin/docs/omd/rapport-omd2009.pdf.

9. Royer M. (1998). Concevoir et naître. Accoucher en Afrique, accoucher en France. Les cahiers de URACA N9. Disponible sur URL: http://uraca-basiliade.org/wpcontent/uploads/2016/12/Uraca_cahier09.pdf.

10. Simkin P. (1990). Just another day in a woman's life? Nature and consistency of women's long-term memories of their first birth. Journal of Psychosomatic Obstetrics and Gynecology; 19(2): 64-81.

11. Szejer M. \& Stewart R. (1994). Ces neuf mois-là, une approche psychanalytique de la grossesse et de la naissance.2eed.Paris: Robert Laffont. Disponiblesururlhttps://www.amazon.fr/Ces-neuf-mois1\%C3\%A0-psychanalytique-grossesse/dp/2221098404(consulté le 13 Mars 2014).

12. Zar M, Wijma K, \& Wijma B. (2001). Pre-and Post partum fear of child birth in Nulliparous and Parous Women. Scandinavian Journal of Behaviour Therapy; 30:75-84. 